\title{
Mesenteric Lymphadenitis
}

National Cancer Institute

\section{Source}

National Cancer Institute. Mesenteric Lymphadenitis. NCI Thesaurus. Code C26830.

Inflammation of the mesenteric lymph nodes. 